X. Ueber die Reflexion des Lichtes an parallet zur optischen Axe geschliffenem Quarz; von Robert Ritter.

(Herza Taf. III Fig. 1-6.)

Auf Anrathen des Brn. Geheimrath v. Helmholtz habe ich mit Hülfe der Wernicke'schen Methode ${ }^{1}$ ) die Reflexion des Lichtes an parallel der optischen Axe geschliffenem Quarz untersucht und gefunden, dass auch bei der Reflexion an diesem doppeltbrechenden Medium nur die senkrecht zur Einfallsebene polarisirte Componente eine Phasenänderung erleidet; ferner war die Wernicke'sche Methode genau genug, um trotz der schwachen Doppelbrechung des Quarzes eine Verschiedenheit des Haupteinfallswinkels je nach der Lage der optischen Axe zur Einfallsebene nachzuweisen. Ich will hier gleich bemerken, dass die Quarzplatte noch mit der vom Polirmittel herrührenden Oberfiächenschicht behaftet war; diese fremdartige Oberflächenschicht übt indessen nur eine modificirende Wirkung aus, wie aus den letzten von Hrn. Wernicke veröffentlichten Versuchen ${ }^{2}$ ) hervorgeht.

Bevor ich zur Beschreibung meiner im hiesigen physikalischen Institute angestellten Versuche übergehe, drängt es mich, meinem hochverehrten Lehrer, Hrn. Geheimrath v. Helmholtz, für die Anregung zu dieser Arbeit, sowie für die Unterstützung und das Interesse, welches er meiner Arbeit stets zu Theil werden liess, meinen ehrerbietigsten Dank zu sagen.

Auch kann ich es nicht unterlassen, an dieser Stelle Hrn. Dr. Wernicke für seine gefälligen Auskünfte und Rathschläge zu danken, welche mir in manchen Einzelheiten von grossem Werth waren.

A. Vorversuch an Glas.

Um mich mit der Methode des Hrn. Wernicke bekannt zu machen, wiederholte ich zunächst seine Versuche an Glas.

1) W. Wernicke, Wied. Ann. 25. p. 203. 1885; 30. p. 452. 1887.

2) W. Wernicke, Wied. Ann. 30. p. 452. 1887. 
Die Luftschicht $z$ wischen zwei zusammengekitteten planparallelen Crownglasplatten zeigte ein Ringsystem, mit einer gleichmässig gefärbten dicksten Stelle in der Mitte, deren Ausdehnung etwa $1 / 2$ qcm betrug, sodass also diese gleichmässig gefärbte Mitte zu den Versuchen verwendet werden konnte. ${ }^{1}$ ) Die zusammengekitteten Platten befestigte ich vertical über der Mitte des Tischchens eines Spectrometers, durch dessen Collimatorrohr ron einem Heliostaten reflectirte Sonnenstrahlen auf die Platten fielen; am Spectrometer wurden die Einfallswinkel gemessen, während zur Bestimmung der Lage der Interferenzstreifen im Spectrum ein selbständiges, schwach vergrösserndes Fernrohr ( $F$, s. Fig. 1 oder Fig. 6) diente, dem ein Prismensystem à vision directe $(G)$ vorgesetzt wurde. Das Fernrohr war mit einem Ocularschraubenmikrometer $M$ versehen, und hinter dem Ocular befand sich ein drehbares Nicol'sches Prisma $N$. - Da es mir anfangs nicht gelang, bei directem Einstellen des Beobachtungsfernrohres $F$ auf den schmalen, im Unendlichen befindlichen Spalt $s_{1}$ die Interferenzstreifen in einem einheitlichen Spectrum mit deutlichen Fraunhofer'schen Linien zu erhalten, so verzichtete ich zunächst auf den Vortheil der Wernicke'schen Methode, den Einfallswinkel ganz genau zu bestimmen, und ordnete beim Versuch mit den Crownglasplatten die Beobachtungsapparate so an, wie es in Fig. 1 abgebildet ist. Diese Anordnung unterscheidet sich von der des Hrn. W ernicke darin, dass ich das ron der Luftschicht reflectirte Licht zunächst durch ein zweites Collimatorrohr $C_{3}$ gehen liess, auf dessen schmalen Spalt $s_{2}$ das selbständige Beobachtungsfernrohr $F$ eingestellt wurde. Dieser schmale Spalt $s_{2}$ ersetzte zugleich den Schirm, welcher das von der ersten Fläche der vorderen und das von der zweiten Fläche der hinteren Glasplatte reflectirte Licht abblenden muss. Um aber bei dieser Anordnung genügende Intensität für die Beobachtung des senkrecht zur Einfallsebene polarisirten Lichtes zu erhalten, musste ich den ersten Collimatorspalt $s_{1}$ breit machen. Bei dem Versuch mit den Crownglasplatten betrug

1) W. Wernicke, Wied. Anu, 25. p. 208, 1885. 
die Spaltbreite desselben 7'. Auf die Platten fiel somit nicht vollständig paralleles Licht, sondern Strahlen, welche untereinander Winkel bildeten, die $\leqq 7^{\prime}$ waren. Die in der Tabelle I angegebenen Einfallswinkel sind somit Mittelwerthe, indem bei Gegenüberstehen ron Collimator $C_{1}$ und Fernrohr $s$ das Mittel aus den Einstellungen auf die beiden Kanten des Collimatorspaltes $s_{1}$ genommen wurde, während die zweite Ablesung durch Fadenkreuzspiegelung an den planparallelen Platten erhalten wurde. Da indessen die in der Tabelle angegebenen Wellenlängen durch Einstellen des Ocularmikrometers auf die Mitte der Interferenzstreifen gefunden wurden, so kann man die angegebenen Einfallswinkel und Wellenlängen als zusammengehörend betrachten. Man sieht übrigens die Berechtigung meiner Anordnung - so lange es sich nur darum handelt, nachzuweisen, dass das parallel der Einfallsebene polarisirte Licht keine Phasenänderung erleidet - leicht ein, wenn man bedenkt, dass bei Einfallswinkeln zwischen 55 und $60^{\circ}$ einem Fehler von $\pm 31_{2}^{\prime}$ in der Bestimmung des Einfallswinkels eine Aenderung der nach der Formel:

$$
d=\frac{m \cdot \lambda}{2 \cdot \cos \alpha}
$$

berechneten Dicke der Luftschicht um 0,0016 - 0,0018 des erhaltenen Werthes $d$ entspricht, dass also selbst der grösste überhaupt mögliche Fehler in der Bestimmung des Einfallswinkels die Zahlen $d$ in der Tabelle I nur um Grössen verändern würde, welche innerhalb der übrigen Beobachtungsfehler liegen und daher keinen Einfluss haben auf die erreichbare Genauigkeit in der Uebereinstimmung der aus verschiedenen Einfallswinkeln berechneten Zahlen $d$. Dementsprechend zeigte sich trotz der grossen Spaltbreite des ersten Collimators keine störende Verbreiterung der Interferenzstreifen.

Die Tabelle I enthält in der ersten Columne die Einfallswinkel $\alpha$, in der zweiten die Ordnungszahl $m$ der Interferenzstreifen, in Columne 3-6: die interferirenden Wellenlängen im parallel und im senkrecht zur Einfallsebene polarisirten Licht, und die aus ihnen nach der Formel: 


$$
d=\frac{m \cdot \lambda}{2 \cdot \cos \alpha}
$$

berechneten Dicken der Luftschicht in $0,0_{5} 1 \mathrm{~mm}$ - während die letzte Columne das Datum der Messung angibt.

Tabelle I.

Luftschicht zwischen Crownglas.

\begin{tabular}{|c|c|c|c|c|c|c|c|c|}
\hline & $\alpha$ & $m$ & $\lambda \|$ & $\lambda 1$ & $d \|$ & $d^{\perp}$ & \multicolumn{2}{|c|}{ Datum für $d \|$} \\
\hline $55^{0}$ & $20^{\prime}$ & $\begin{array}{l}3 \\
4\end{array}$ & $\begin{array}{l}561,6 \\
422,2\end{array}$ & 574,1 & $\begin{array}{l}1481 \\
1484\end{array}$ & 1514 & 19. Juli & 1886 \\
\hline 56 & - & $\begin{array}{l}3 \\
4\end{array}$ & $\begin{array}{l}550,7 \\
413,6\end{array}$ & 581,5 & $\begin{array}{l}1477 \\
1479\end{array}$ & 1560 & $\begin{array}{l}26 . \quad " \\
30 .\end{array}$ & $"$ \\
\hline 56 & 15 & $\begin{array}{l}3 \\
4\end{array}$ & $\begin{array}{l}549,7 \\
411,7\end{array}$ & 590,2 & $\begin{array}{l}1484 \\
1482\end{array}$ & 1593 & 6. Aug. & $"$ \\
\hline 56 & 30 & $\begin{array}{l}3 \\
4\end{array}$ & $\begin{array}{l}543,7 \\
409,5\end{array}$ & 639,5 & $\begin{array}{l}1478 \\
1484\end{array}$ & 1738 & 9. & $"$ \\
\hline 56 & 45 & $\begin{array}{l}3 \\
4\end{array}$ & $\begin{array}{l}540,2 \\
405,9\end{array}$ & & $\begin{array}{l}1478 \\
1481\end{array}$ & & $9 . \quad "$ & $"$ \\
\hline 57 & - & $\begin{array}{l}3 \\
4 \\
3\end{array}$ & $\begin{array}{l}535,6 \\
534,1\end{array}$ & 505,4 & $\begin{array}{l}1475 \\
1471\end{array}$ & 1856 & 10. " & $"$ \\
\hline 57 & 30 & 3 & 527,1 & & 1471 & & & \\
\hline 58 & 30 & $\begin{array}{l}4 \\
3 \\
4\end{array}$ & 513,2 & $\begin{array}{l}508,3 \\
503,9\end{array}$ & 1473 & $\begin{array}{l}1892 \\
1929\end{array}$ & 13. $"$ & $"$ \\
\hline
\end{tabular}

Die Interferenzstreifen waren wegen der Form der Luftschicht nach dem rothen Ende des Spectrums hin convex, und wurde immer auf die äusserst zum rothen Ende hin gelegene, den Fraunhofer'schen Linien parallele Stelle derselben eingestellt. Die Platten waren noch mit der vom Polirmittel herrührenden Oberflächenschicht behaftet. Die Wellenlängen der Streifen im senkrecht zur Einfallsebene polarisirten Licht sind zum Theil nur geschätzt; sie dürfen daher nicht zu Messungen der Phasendifferenzen verwendet werden.

Die in Tabelle I angegehenen Zahlen sind - ungünstiger Witterung wegen, und weil ich zum schnellen Beobachten noch nicht geübt genug war - im Laufe von etwa drei Wochen (Ende Juli und Anfang August 1886) gemessen worden; trotzdem stimmen die im parallel zur Einfallsebene polarisirten Licht erhaltenen Zahlen $d^{\|}$für die Dicke der Luftschicht gut überein - ein Zeichen für die grosse Unempindlichkeit der Kittbefestigung gegen Temperatureinflüsse. 
B. Versuche an parallel zur optischen Axe geschliffenem Quarz.

1. Schraubenapparat. - Um die Reflexion des Lichtes an parallel zur optischen Axe geschliffenem Quarz mit Hülfe der Wernicke'schen Methode zu untersuchen, musste die hintere Glasplatte durch eine Quarzplatte ersetzt werden, d. h. die Versuche mussten an einer Luftschicht zwischen Glas und Quarz angestellt werden. Um diese Luftschicht möglichst gleichmässig machen zu können und zugleich im Stande zu sein, dieselbe ie nach dem $Z$ wecke des betreffenden Versuchs nach Wunsch bald dicker, bald dünner zu erhalten, wurde nach der Idee des Hrn. Geheimraths v. Helmholtz in der mechanischen Werkstatt von Franz Schmidt und Hänsch ein Schraubenapparat hergestellt, dessen Totalansicht Fig, 2 zeigt, während in Fig. 3 sein verticaler Durchschnitt, in Fig. 4 und 5 seine beiden Haupttheile abgebildet sind. Zunüchst wurde eine parallel zur Axe geschliffene, quadratische $(4,4 \mathrm{~cm})^{2}=19,36 \mathrm{qcm}$ grosse Bergkrystallplatte speciell gegen eine ebenso grosse und ebenso geformte planparallele Crownglasplatte so abgeschliffen, dass man beim Aufeinanderlegen der beiden Platten mit Leich. tigkeit eine in ihrer ganzen Ausdehnung gleichmässig gefärbte Luftschicht erbielt. Hierauf wurde jede Platte in eine Messingfassung $M$ (s. Fig. 2, 4 und 5) gekittet, und zwar so, dass die etwa $8 \mathrm{~mm}$ dicken Platten auf beiden Seiten der etwas dünneren Fassung ein wenig herrorragen, sodass auch jetzt noch die beiden gegen einander abgeschliffenen Flächen in Berührung gebracht werden können wenn die acht in der Fassung der Glasplatte angebrachten Schraubenfedern, deren Köpfe $f$ in Fig. 2, 3 und 4 sichtbar sind, ausser Thätigkeit gesetzt sind. - Fig. 2 und 4 zeigen uns die äussere Seite der F'assung der Glasplatte. Die Köpfe der Federn sind durch einen Rahmen $R$ hindurchgeführt, welcher vermittelst der gegen die Fassung drückenden Schraube $F$ schlittenartig gegen dieselbe verschoben werden kann. Hat der Rahmen $\boldsymbol{R}$ die Stellung der Fig. 2, so ragen die Enden der Federn auf der inneren Seite der Fassung 
über die Glasplatte hervor, wie man es in Fig. 3 angedeutet sieht, wird er dagegen vermittelst der Schraube $F$ nach oben verschoben, sodass er die Lage der Fig. 4 erbält, so fassen die auf dem Rahmen befindlichen keilförmigen Erhöhungen $k$ die benachbarten Köpfe der Federn $f$, heben dieselben in die Höhe und setzen auf diese $W$ eise die Federn ausser Thätigkeit, indem dann die Enden derselben auf der inneren Seite der Fassung nicht mehr über die Glasplatte hervorragen, sodass man jetzt die Quarzplatte mit der Glasplatte in Berührung bringen kann. Nachdem man die Federn $f$ auf diese Weise ausser Thätigkeit gesetzt hat, legt man die eingefasste Quarzplatte auf die "innere", in Fig. 4 nicht sichtbare Seite der Glasplatte, sodass die beiden Platten mit ihren ähnlichen Fassungen in übereinstimmender Stellung übereinander liegen. Waren beide vorher sorgfältig gereinigt, so erhält man auch jetzt mit Leichtigkeit eine rollkommen gleichmässig gefärbte Luftschicht, da die Platten trotz des Einkittens in die Messingfassungen ihre vorzüglich ebenen Flächen behalten hatten. Hierauf werden die an den vier Ecken der Glasplattenfassung angebrachten Scharnire $n$ (s. Fig. 4), welche die Schrauben $s$ tragen, in die Höhe gehoben und durch die vier Schrauben $s$ die beiden Platten mit schwachem Druck zusammengehalten. Wird hierauf der Rahmen $R$ wieder in seine in der Fig. 2 angegebene Stellung gebracht, so sind die Federn $f$ in Thätigkeit und haben das Bestreben, die beiden Platten möglichst voneinander zu entfernen, während dieselben von den vier Schrauben $s$ zusammengehalten werden. Hierdurch ist es ermöglicht, der Luftschicht auch bei verticaler Stellung der Platten durch Verstellen der vier Schrauben $s$ jede gewünschte Dicke zu ertheilen, indem die Luftschicht wegen der Federn $f$ jedesmal die grösste von den Schrauben $s$ gestattete Dicke annimmt. Die Symmetrie der Lage der Federn $f$ trägt wohl dazu bei, dass zugleich auf diese Weise die Dicke der Luftschicht, wie wir sehen werden, sehr gleichmässig gemacht werden kann.

Die Totalansicht des Schraubenapparates (Fig. 2) und der verticale Durchschnitt (Fig. 3) zeigen, wie die beiden in der beschriebenen Weise zusammengehaltenen Platten über Ann. d. Phys. u. Chem. N. F. XXXVI. 
dem Spectrometertischchen angebracht werden. Die Kreisscheibe $T$ wird auf das Tischchen gesetzt und vermittelst der beiden Schrauben $t$ an demselben befestigt. Sie trägt ein Ansatzstück $A$, in welchem eine Schraubenspindel $P$ sich drehen lässt. In der Fassung der Quarzplatte befanden sich zwei Oeffnungen ( $a, b$ Fig. 5) mit Gewinden; diente die eine als Schraubenmutter, so war die optische Axe des Krystalls horizontal; diente die andere Oeffnung als Schraubenmutter, so war sie vertical, stand also senkrecht zur Einfallsebene. Das Ansatzstück $A$ war so angebracht, dass die Luftschicht zwischen den beiden Platten über der Axo des Spectrometers zu stehen kam.

Das von mir benutzte Spectrometer - ein alter, sehr schöner Apparat ron Pistor und Martins - gestattete eine horizontale Verschiebung des Tischchens von je $1 \mathrm{~cm}$ in zwei zu einander senkrechten Richtungen, deren eine der Ebene der Platten parallel war; dies war ein grosser Vortheil für meine Versuche, indem ich auf solche $W$ eise die Möglichkeit hatte, auch bei dickeren Luftschichten, wo die Newton'schen Farben nicht mehr zu erkennen sind, und überhaupt viel genauer, als es vermittelst der $\mathrm{New}$ to n'schen Farben möglich ist, die Gleichmässigkeit der Luftschicht zu prüfen, resp. durch Verstellen der Schrauben $s$ zu verbessern. Betrachte ich nämlich die von der Luftschicht herrührenden Interferenzstreifen im parallel der Einfallsebene polarisirten Licht und verschiebe dabei die Luftschicht in ihrer eigenen Ebene, so werden die Interferenzstreifen nur in dem Fall ihre Lage im Spectrum unverändert beibehalten, dass die Luftschicht an allen bei der Verschiebung nach und nach beobachteten Stellen die gleiche Dicke hat, dagegen bewirkt auch die kleinste Ungleichmässigkeit der Dicke der Luftschicht eine Verschiebung der Interferenzstreifen; sie bleiben dabei parallel den Fraunhofer'schen Linien, wenn die Luftschicht nur in horizontaler Richtung ungleichmässig dick ist, während eine Ungleichmässigkeit in verticaler Richtung eine Neigung oder Krümmung der Streifen hervorruft. So kann man sofort die Gestalt der Luftschicht beurtheilen und weiss, welche der vier Schrauben $s$, und wie man sie zu ver- 
stellen hat, um die Dicke der Luftschicht gleichmässiger zu machen. Auf diese Weise kann man die Luftschicht viel gleichmässiger machen, als mit Hülfe des Kriteriums der überall gleichen Newton'schen Farbe; sehr häufig erschien die Luftschicht in ihrer ganzen Ausdehnung - mit freiem Auge subjectiv betrachtet - gleichmässig gefärbt, während die Interferenzstreifen bei einer Verschiebung der Platten merklich wanderten. Bei einiger Ausdauer gelingt es indessen, durch Verstellen der vier Schrauben $s$ dies Wandern zu vermeiden oder auf ein Minimum zu beschränken und auf diese $W$ eise die Luftschicht in einer Ausdehnung von etwa $1 \mathrm{qcm}$ vollkommen gleichmässig dick zu machen.

Die Möglichkeit der Verschiebung des Spectrometertischchens war ein Ersatz für einen empfindlichen Mangel, der leider einem solchen Schraubenapparat im Gegensatz zur einfachen Kittbefestigung anhaftet, und der in der grossen Empfindlichkeit für Temperatureinflüsse besteht. Wir sahen aus Tabelle I, wie bei einer solchen Kittbefestigung die Luftschicht während mehrerer Wochen eine nahezu constante Dicke behielt, trotzdem bei jenen Versuchen keine irgendwie bedentenden Schutzmaassregeln gegen Temperatureinflüsse getroffen worden waren. Beim Schraubenapparat kann sich die Dieke der Luftschicht schon während weniger Minuten sehr bedeutend ändern, wenn nicht sorgfältige Vorkehrungen gegen Temperatureinflüsse getrotfen sind, wenn vor allem nicht jede Spur von strahlender Wärme abgehalten wird. Aber selbst bei grösster Vorsicht ist es nicht immer möglich, Temperaturschwankungen völlig zu vermeiden; so zeigt sich z. B. an heissen, wolkenlosen Sommertagen, die andererseits gerade am günstigsten für diese Versuche sind, fast immer mit dem Ansteigen der Temperatur während des Tages ein Dickerwerden der Luftschicht.

Ich werde bei Besprechung der Resultate der einzelnen Versuche auf diese Temperatureinflüsse zurückkommen. Es wird sich zeigen, dass der beschriebene Schraubenapparat besonders gut zu Messungen der Phasendifferenzen sich eignet, wobei die Temperaturänderungen nur wenig in Betracht kommen und auf einfache Weise eliminirt werden können. 
2. Bestimmung der Richtung der optischen Axe. - Da ich die Quarzplatte eingefasst und ohne Angabe der Richtung der optischen Axe in der geschliffenen Fläche aus der mechanischen Werkstatt erhalten hatte, so musste ich diese Richtung auf optischem Wege bestimmen.

Hierzu brachte ich die Platte zunächst zwischen zwei gekreuzte Nicol'sche Prismen und erhielt aus der Lage der Platte, bei welcher keine Depolarisation eintritt, die beiden aufeinander senkrechten Richtungen, von denen die eine die Richtung der optischen Axe ist. Eine dieser Richtungen wurde auf der Fassung der Quarzplatte markirt. Um zu entscheiden, welche der zwei zu einander senkrechten Richtungen die Richtung der Axe ist, bestimmte ich am Spectrometer den Winkel, welchen die beiden Ebenen der schwach prismatischen Quarzplatte bilden, und die Lage der Kante des Prismas und wusste somit, an welcher Seite die Platte dicker, und an welcher Seite sie dünner war. Die Richtung senkrecht zur Prismenkante wurde auf der Fassung markirt, und zwar als Pfeil, welcher nach der dünnen Seite der Quarzplatte hinweist (s. Fig. 5). Hierauf untersuchte ich die Quarzplatte in einem mikroskopischen Polarisationsapparat, welcher das auf die Platte fallende Licht sehr convergent machte. Bei Anwendung von homogenem Na-Licht zeigte die etwa $8 \mathrm{~mm}$ dicke Platte zwei Hyperbelsysteme, deren aufeinander senkrechte Axen der Richtung der optischen Axe und der ihr senkrechten Richtung entsprachen. Der von den beiden Ebenen der Quarzplatte eingeschlossene Winkel betrug $10^{\prime} 20^{\prime \prime}$, die Ausdehnung der Platte in der Richtung senkrecht zur Prismenkante $55 \mathrm{~mm}$, somit der Unterschied der. Dicke an den beiden Enden der Platte in dieser Richtung: $55 . \sin \cdot 10^{\prime} 20^{\prime \prime}=55.0,003=0,165 \mathrm{~mm}$. Einem Gangunterschied der zwei den Quarz in normaler Richtung durchsetzenden Strahlen von einer halben Wellenlänge entspricht eine Dicke von $0,03237 \mathrm{~mm}$; bei Verschiebung der Platte im Polarisationsapparat in Richtung senkrecht zur Prismenkante musste somit die Mitte des Gesichtsfeldes die Intensität wechseln; war die Mitte anfangs gelb, so wurde sie allmählich schwarz, dann wieder gelb u. s. f., 
d. h. sie wechselte fünfmal die Intensität, weil der Dickenunterschied von $0,165 \mathrm{~mm}$ einer Aenderung der Phasendifferenz der beiden Strahlen von ungefähr fünf halben Wellenlängen entspricht.

Dabei näherten sich die Hyperbeln des einen Systems der Mitte des Gesichtsfeldes und den Asymptoten, in welchen sie schliesslich verschwandẹn, während die Hyperbeln des anderen Systems sich von der Mitte und den Asymptoten entfernten, und von den Asymptoten her immer neue Hyberbeln dieses Systems auftraten.

Wenn ich die Platte so verschiebe, dass zuerst die dicken, dann die dünnen Stellen untersucht werden, so ist die optische Axe der Axe jenes Systems parallel, dessen Hyperbeln sich der Mitte nähern; denn in ihrer Richtung nimmt der Gangunterschied der interferirenden Strahlen bei wachsender Entfernung von der Mitte des Gesichtsfeldes ab. - Auf diese Weise ergab sich, dass die auf der Fassung markirte Richtung die Richtung senkrecht zur optischen Axe ist.

3. Beobachtungen unter beliebigen Einfallswinkeln. - Zunächst habe ich zwei Versuche (Tabelle II und III) bei parallel zur Einfallsebene polarisirtem Licht gemacht, um nachzuweisen, dass bei der Reflexion an Quarz unter beliebigen Einfallswinkeln, ebenso wie bei der Reflexion an isotropen durchsichtigen Medien, die parallel zur Einfallsebene polarisirte Componente keine Phasenänderung erleidet. Bei dem einen Versuch stand die optische Axe des Quarzes senkrecht, beim anderen parallel zur Einfallsebene. Ich konnte hierbei wieder die Anordnung der Apparate, wie sie in der Fig. 1 dargestellt ist, anwenden. Die Spaltbreite des ersten Collimators $C_{1}$ betrug $4^{\prime}$, resp. 5'. Aber selbst ein Feller von $\pm 2^{1} /_{2}^{\prime}$ in der Bestimmung des Einfallswinkels würde die nach der Formel:

$$
d=\frac{m \cdot \lambda}{2 \cdot \cos \cdot \alpha}
$$

berechnete Dicke der Luftschicht bei Einfallswinkeln von $40-65^{\circ}$ nur um $0,0_{3} 6-0,0_{2} 16$ des Gesammtbetrages verändern - Grössen, welche nicht in Betracht kommen. 
Zur Bestimmung der Einfallswinkel wurde zunächst ohne dazwischenstehende Platten die Gegenüberstellung von Collimator- und Fernrobr abgelesen, alsdann der Schraubenapparat an das Ansatzstück über dem Spectrometertischchen angeschraubt, die senkrecht zur optischen Axe der Quarzplatte stehende Marke an der Fassung derselben horizontal, resp. vertical gestellt und hierauf die planparallele Glasplatte und mit ihr die vordere Fläche der schwach prismatischen Quarzplatte durch Fadenkreuzspiegelung und durch Verstellen der unter dem Spectrometertischchen dazu vorhandenen Schrauben parallel zur Umdrelungsaxe des Spectrometers, also vertical gestellt. Die zweite Einstellung zur Bestimmung des Einfallswinkels geschah durch Einstellen des Spectrometerfernrohres $s$ auf die Mitte des von der planparallelen Glasplatte gespiegelten Spaltes, weil dies die schnellste Einstellung ist. Hierauf wurde das Spectrometerfernrohr $S$ zur Seite gedreht, das zweite Collimatorrohr $C_{2}$ und das Beobachtungsfernrohr $F$ mit Prismensystem für gerade Durchsicht an seine Stelle gebracht (s. Fig. 1), und mit der Messung der Interferenzstreifen begonnen. Nach beendigter Messung wurde zur Controle nochmals das Spectrometerfernrohr $S$ auf die Mitte des von der planparallelen Glasplatte reflectirten Spaltes eingestellt.

Zu Anfang eines jeden der beiden Versuche war die Luftschicht zwischen den Platten möglichst gleichmässig gemacht worden durch horizontales Verschieben derselben in ihrer eigenen Ebene und durch Verstellen der Schrauben $s$ (s. Fig. 2, 3, 4), bis die Interferenzstreifen bei einer solchen Verschiebung der Platten nicht mehr oder nur ganz unbedeutend ihre Lage im Spectrum veränderten. Hierauf wurden während eines jeden Versuchs die Schrauben $s$ nicht mehr berührt, also die Dicke der Luftschicht - bis auf Temperatureinflüsse - unverändert gelassen. Man darf daher alle Zahlen $d$, welche in der Tabelle II, resp. III angegeben sind, untereinander vergleichen. Doch wird man es begreiflich finden, dass die Zahlen $d$, welche an einem Tage beobachtet sind, besser untereinander übereinstimmen, als sie es mit an anderen Tagen gefundenen thun. Dass die vorkom- 
menden geringen Verschiedenheiten der Zahlen $d$ nicht auf Phasenänderungen beruhen, sieht man schon daraus, dass die absichtlich wiederholten Messungen unter einem schon früher untersuchten Einfallswinkel dieselben Abweichungen gegen die früher unter diesem Einfallswinkel gefundenen Zahlen zeigen, wie die Zahlen verschiedener Einfallswinkel. Die Verschiedenheiten der Zahlen $d$ sind, sofern sie nicht in Beobachtungsfehlern ihren Grund haben, die Folge von wirklichen Veränderungen der Dicke und Form der Luftschicht, welche durch Temperaturänderungen oder durch eine elastische Nachwirkung der Federn $f$ hervorgerufen werden.

So scheint die grösste Abweichung, die der Zahlen $d$ vom 14. Februar gegen die Zahlen der folgenden Tage, die Folge einer Nachwirkung der Federn $f$ gewesen zu sein, welche am ersten Tage, an welchem die Schrauben $s$ eingestellt worden waren, noch nicht zur vollen Geltung gekommen sein mögen. Denn die Luftschicht war am folgenden Tage dicker, trotzdem die Zimmertemperatur um $1,7^{\circ} \mathrm{C}$. gefallen war. Dass aber die Dicke der Luftschicht $z$ wischen den Platten unter dem blossen Einfluss der Temperatur zugleich mit der Temperatur wächst und abnimmt, tritt in den späteren Versuchen, welche ich im Sommer bei weit höherer Temperatur angestellt habe, deutlich hervor und zeigt sich ebenso in den Zahlen vom 17. Februar, welche ich erhalten habe, nachdem ich durch Oeffnen der Luftheizungsklappe die Temperatur absichtlich erhöht hatte. Doch kann man die Temperatur nicht einfach in Rechnung bringen, weil die Verhältnisse der Messingfassung und der glatten Obertächen der eingefassten Platten zu complicirt sind, und man nicht annehmen kann, dass alle Theile des Schraubenapparates zu gleicher Zeit und sofort die Temperatur der den Apparat umgebenden Luft annehmen, welche in den Tabellen ange. geben ist; ferner machen die Temperaturänderungen die Luftschicht nicht einfach dicker oder dünner, sondern verändern in unregelmässiger Weise ihre Form, indem sie auf die einzelnen Schrauben und Federn in unregelmässiger Weise einwirken, wodurch die Luftschicht gewöhnlich un- 
gleichmässiger wird. Es bleibt daher nur übrig, die Temperatur während des Versuchs möglichst constant zu erhalten und die Messungen möglichst schnell auszuführen, um eine grössere Anzahl Zahlen in verhältnissmässig kurzer Zeit zur Vergleichung zu erhalten. Die angegebenen Wellenlängen sind daher das Mittel aus nur drei, übrigens gut übereinstimmenden Mikrometereinstellungen. Sie sind in beiden Tabellen in der Reihenfolge angegeben, in welcher sie gemessen worden sind. Die mit ${ }^{*}$ bezeichneten Zahlen sind nur geschätzt.

Tabelle II.

Luftschicht zwischen Glas und Quarz im parallel der Eiufallsebene polarisirten Licht.

Optische Axe senkrecht zur Einfallsebene.

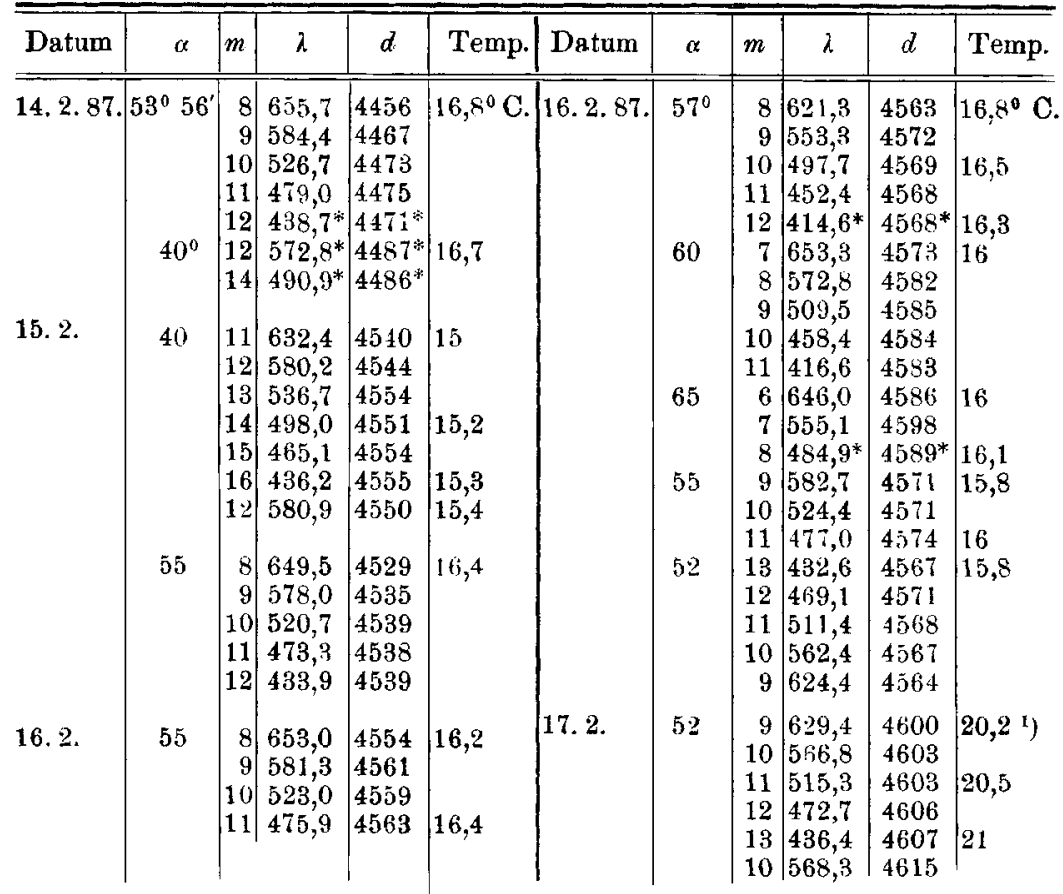

1) Bei offener Iuftheizungsklappe. 
Tabelle III.

Luftschicht zwischen Glas und Quarz im parallel zur Einfallsebene polarisirten Licht.

Optische Axe parallel der Einfallsebene.

\begin{tabular}{l|c|c|c|c|c|c|c|c|c|c|c}
\hline \hline Datum & $\alpha$ & $m$ & 2 & $d$ & Temp. & Datum & $\alpha$ & $m$ & $\lambda$ & $d$ & Temp. \\
\hline \hline 11.3.87. $54^{0}$ & 10 & 674,0 & 5733 & $18^{\circ} \mathrm{C}$. & 13.3 .87$. & $50^{\circ}$ & 11 & 665,3 & 5693 & $19^{\circ} \mathrm{C}$. \\
& & 11 & 614,7 & 5752 & & & & 12 & 611,2 & 5705 & \\
& & 12 & 563,3 & 5750 & & & & 13 & 564,1 & 5704 & \\
& & 13 & 519,9 & 5749 & & & & 14 & 523,3 & 5699 & \\
& & 14 & 482,8 & 5750 & & & & 15 & 488,3 & 5697 & \\
& & 15 & 450,6 & 5749 & & & & 16 & 457,5 & 5694 & \\
& 16 & 422,4 & 5749 & & & & 17 & 430,3 & 5690 & \\
& 57 & 15 & 415,5 & 5722 & 20 & & & & & & \\
& 14 & 445,7 & 5729 & & & 40 & 13 & 670,6 & 5690 & 20 \\
& 13 & 480,0 & 5729 & & & & 14 & 623,4 & 5697 & \\
& 12 & 519,6 & 5725 & & & & 15 & 582,3 & 5701 & \\
& 11 & 567,6 & 5732 & & & & 15 & 583,5 & 5713 & 20,4 \\
& 10 & 623,2 & 5722 & 19,6 & & & 16 & 547,9 & 5722 & \\
& 59 & 10 & 589,1 & 5719 & & & & 17 & 514,3 & 5707 & \\
& 11 & 536,1 & 5725 & & & & 18 & 486,0 & 5710 & \\
& 12 & 490,7 & 5717 & & & & 19 & 460,7 & 5714 & \\
& 13 & 453,2 & 5720 & 20 & & & 20 & 437,6 & 5713 & \\
& 50 & 12 & 612,3 & 5715 & 20,6 & & & 21 & 416,7 & 5712 & \\
& 13 & 565,8 & 5721 & & & & & & & \\
& 14 & 525,6 & 5724 & & & & & & & \\
& 15 & 490,5 & 5723 & & & & & & &
\end{tabular}

Ausser der directen Wirkung von Temperaturänderungen und elastischer Nachwirkung müssen die Beobachtungsfehler erwähnt werden, welche daraus entstanden, dass nicht immer ganz dieselbe Stelle der Luftschicht und hierdurch, nachdem die Luftschicht durch Temperatureinflüsse und elastische Nachwirkung etwas ungleichmässig geworden war, verschieden dicke Stellen der Luftschicht zur Untersuchung kamen. So zeigt sich z. B. an den Zahlen des 16. Februar das Folgende: das Sonnenbildchen auf der Linse des ersten Collimators $C_{\mathbf{1}}$ wurde immer genau auf derselben Stelle erhalten; würden die Strahlen in gerader Linie bis zur Luftschicht gelangen, so würden sie also stets dieselbe Stelle der Luftschicht treffen; da sie aber bei verschiedenen Einfallswinkeln versehieden starke Ablenkung in der planparallelen Glasplatte erleiden, so geschieht dies nicht. Bei grösseren Einfallswinkeln $\left(\alpha=65^{\circ}\right)$ wurden dickere Stellen getroffen, als 
bei kleineren. Daher der Verlauf der Zahlen $d$ am 16. Februar. - Beim Einfallswinkel $\alpha=40^{\circ}$ am 15. Februar war dieser Fehler durch eine kleine Verschiebung des Tischchens compensirt worden. - Die hieraus entstandenen Abweichungen verdecken in den Tabellen II und III meistentheils die Richtung der Temperatureinflüsse. Bei den späteren, in Tabelle IV, V und VI mitgetheilten Versuchen fallen die von der Ablenkung in der planparallelen Glasplatte herrührenden Fehler fort, weil dort die Einfallswinkel nur wenig verändert wurden.

4. Beobachtungen in der Nähe des Haupteinfallswinkels. - Bei den bisher mitgetheilten Versuchen konnte die in Fig. 1 dargestellte Anordnung der Apparate angewandt werden, da es sich in diesen Versuchen nur um qualitative Untersuchungen handelte. Will man aber die in der Nähe des Haupteinfallswinkels erhaltenen Zahlen zu Messungen der absoluten Phasenänderungen der senkrecht zur Einfallsebene polarisirten Componente (Phasendifferenzen der beiden Componenten) verwenden und hieraus den Haupteinfallswinkel berechnen, so ist eine vollkommen genaue Bestimmung des Einfallswinkels unbedingt nothwendig, da bekanntlich die Phasendifferenzen äusserst schnell sich mit dem Einfallswinkel ändern. Ich habe deshalb bei allen folgenden Versuchen das zweite Collimatorrohr $C_{2}$ beseitigt (s. Fig. 6) und das Beobachtungsfernrohr $F$ auf den schmalen Collimatorspalt $s_{1}$ eingestellt, welcher so breit war, dass er gerade noch deutliche Fraunhofer'sche Linien lieferte. Die Einfallswinkel wurden in ähnlicher Weise wie für Tabelle II und III vermittelst des Spectrometerfernrohrs $S$ gemessen. Sie sind bis auf $\pm 30^{\prime \prime}$ genau. Das Spectrometer erlaubte zwar, die Einfallswinkel noch viel genauer zu messen, allein es kam darauf an, möglichst schnell zu beobachten, um eine möglichst grosse Anzahl Zahlen zur Vergleichung zu erhalten. Daher wurde auch nur an einem der beiden mit dem Spectrometerfernrohr $S$ fest verbundenen Mikroskope $m$ abgelesen, sodass die Excentricität des Spectrometers beim möglichen Fehler in Rechnung gezogen werden musste.

Die Tabellen IV und $V$ geben die bei verschiedenen 
Einfallswinkeln $a$ gemessenen Wellenlängen, $\lambda \|$ und $\lambda \perp$, der Interferenzstreifen im parallel and senkrecht polarisirten Licht (Columne 3 und 4), und die aus denselben nach der Formel:

$$
d=\frac{m \cdot \lambda}{2 \cdot \cos \cdot \alpha}
$$

berechneten Grössen $d^{\|}$and $d \perp$ (Columne 5 und 6) in Milliontelmillimetern. Columne 7 gibt die Zimmertemperatur $t$ in Celsiusgraden an. Es dürfen in diesen Tabellen nur die Zahlen $d \|$ und $d \perp$, welche an einem und demselben Tage beobachtet worden sind, verglichen werden, da zwischen den einzelnen Beobachtungstagen die Schrauben $s$ (Fig. 2, 3, 4) verstellt und somit die Dicke der Luftschicht verändert worden war. Die Wellenlängen $\lambda \|$ sind das Mittel aus drei Einstellungen, die Wellenlängen $\lambda \perp \mathrm{im}$ allgemeinen das Mittel aus fünf Einstellungen des Ocularmikrometers $M$. Die Zahlen $\lambda !$ und $d^{\prime \prime}$ sind innerhalb eines jeden Beobachtungstages in der Reihenfolge angegeben, in welcher sie gemessen worden sind.

\section{Tabelle IV.}

Luftschicht zwischen Glas und Quarz.

Optische Axe senkrecht zur Einfallsebene.

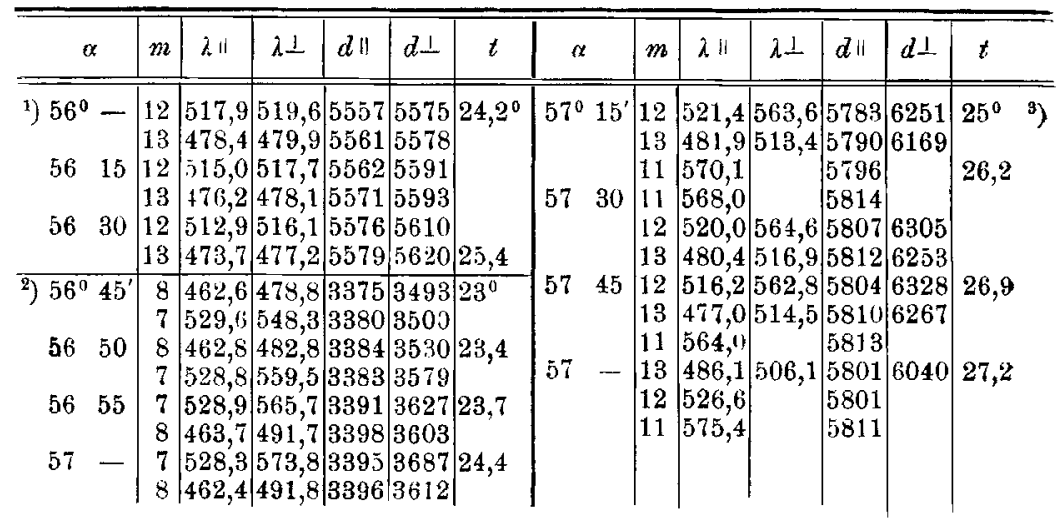

1) am 22.7.87, 2) am 25.6, 3) am 14.7 beobachtet. 
Tabelle V.

Luftschicht zwischen Glas und Quarz.

Optische Axe parallel der Einfallsebene.

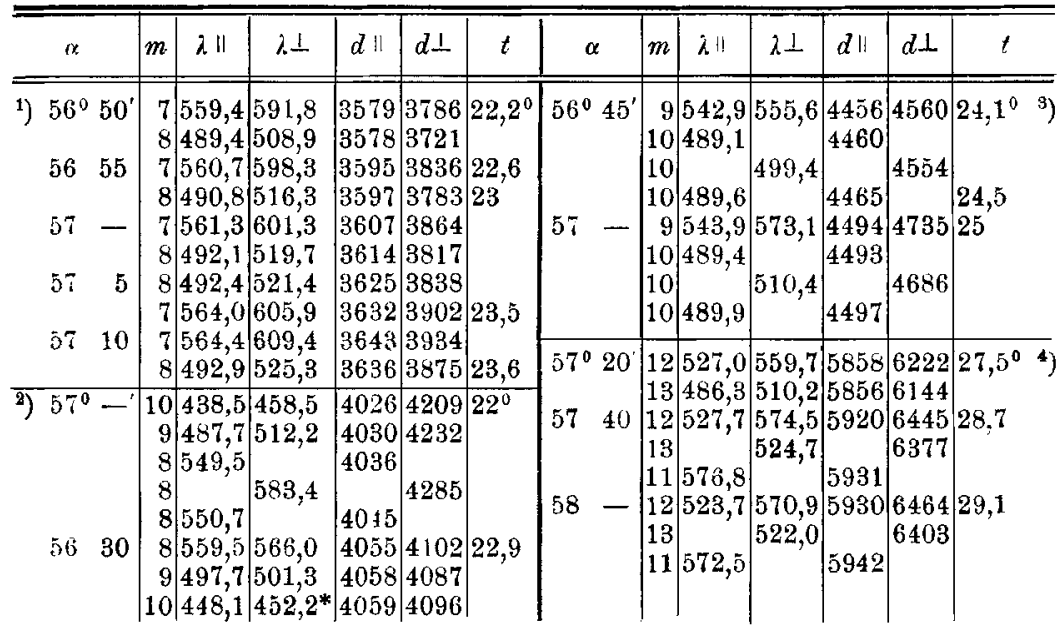

Man ersieht aus diesen beiden Tabellen, dass das parallel der Einfallsebene polarisirte Licht auch in der Nähe des Haupteinfallswinkels ohne Phasenänderung reflectirt wird, und dass die mit dem Babinet'schen Compensator beob. achteten Phasendifferenzen nur von einer Phasenänderung der senkrecht polarisirten Componente herrühren. - Solange das Nicol'sche Prisma nur eine Spur von parallel polarisirtem Licht durchliess, hatten die Interferenzstreifen die ihnen in diesem Lichte zukommende Lage im Spectrum, und erst im Augenblick, wo das Nicol die Stellung einnimmt, in welcher es nur senkrecht polarisirtes Licht hindurch lässt, tritt ein plötzliches Ueberspringen der Streifen in ihre neue Lage ein, was einen sehr interessanten Anblick gewährt.

Das von der vorderen Glasfläche und von der hinteren Quarzfläche reflectirte Licht wurde durch den Schirm $r$ (s. Fig. 6) abgeblendet, dessen verticaler Spalt etwa $1 \mathrm{~mm}$ breit war; trotzdem waren die Interferenzstreifen bei gleichmässiger Luftschicht in beiden Lichtarten vollkommen schwarz, und konnte dies als nothwendiges Kriterium für

1) am 27.6.; 2) am 29.6.; 3) am 4.7.; 4) am 29.7. beob. 
die Gültigkeit der Versuche dienen: die Luftschicht musste mindestens so gleichmässig sein, dass die Streifen vollkommen schwarz erschienen. Die Interferenzen im senkrecht polarisirten Licht hatten gewöhnlich die Form ron schwarzen Flecken, da der obere und untere Theil des Gesichtsfeldes noch von parallel polarisirtem Licht erleuchtet wurde.

Das geringe Wachsen der Zahlen $d^{\|}$- hauptsächlich in der Tab. V - ist offenbar die Folge des im Laufe des Tags eintretenden Temperaturanstieges, welcher sich gerade an völlig wolkenlosen und daher heissen Sommertagen am schwierigsten vermeiden lässt. In Tab. VI theile ich einen Versuch vom 30. Juli 1887 mit, bei welchem die optische Axe parallel der Einfallsebene war, und bei welchem ich nur die Interferenzstreifen im parallel polarisirten Licht gemessen habe. Dieser Versuch zeigt freilich auch ein allmähliches Ansteigen der Zahlen $d^{\|}$, doch kann er trotzdem als Ergänzung der Tab. $V$ dienen, wenn man z. B. in der letzteren die Zahlen vom 29. Juni und vom 29. Juli mit den Zahlen der entsprechenden Einfallswinkel in der Tab. VI vergleicht. Bei diesem Versuch wurden die Streifen während des Tags etwas geneigt gegen die Fraunhofer'schen Linien, dafür blieb aber die Luftschicht in horizontaler Richtung recht gleichmässig; und es wurde genau darauf geachtet, dass immer dieselbe Stelle der Luftschicht untersucht wurde.

\section{Tabelle VI.}

Luftschicht zwischen Glas und Quarz im parallel zur Einfallsebene polarisirten Licht.

Optische Axe parallel der Einfallsebene.

\begin{tabular}{c|c|c|c|c|c|c|c|c|c}
\hline \hline$\alpha$ & $m$ & $\lambda \|$ & $d \|$ & $t$ & $\alpha$ & $m$ & $\lambda \|$ & $a^{3} \|$ & $t$ \\
\hline \hline $56^{\circ} 30^{\prime}$ & 10 & 642,2 & 5818 & $28,5^{\circ}$ & $56^{0} 45^{\prime}$ & 10 & 641,5 & 5850 & \\
& 11 & 583,7 & 5816 & & & 11 & 582,5 & 5843 & \\
& 12 & 534,6 & 5812 & & & 12 & 534,0 & 5844 & \\
& 13 & 493,4 & 5811 & & & 13 & 492,7 & 5841 & \\
& 14 & 459,1 & 5823 & & & 14 & 458,1 & 5849 & \\
& 15 & 428,6 & 5824 & & & 15 & 427,5 & 5848 & \\
& 16 & 402,0 & 5827 & & & 16 & 400,6 & 5845 &
\end{tabular}




\begin{tabular}{|c|c|c|c|c|c|c|c|c|c|}
\hline$\alpha$ & $m$ & $\lambda \|$ & $d \|$ & $t$ & $\alpha$ & $m$ & $\lambda \|$ & $d \|$ & $t$ \\
\hline $57^{\circ}-$ & $\begin{array}{l}10 \\
11 \\
12 \\
13 \\
14 \\
15 \\
16\end{array}$ & $\begin{array}{l}639,4 \\
581,6 \\
532,8 \\
491,8 \\
457,8 \\
427,1 \\
400,5\end{array}$ & $\begin{array}{l}5870 \\
5873 \\
5870 \\
5869 \\
5884 \\
5881 \\
58 \times 3\end{array}$ & $29,2^{0}$ & $57 \quad 45$ & $\begin{array}{l}10 \\
11 \\
12 \\
13 \\
14 \\
10 \\
11\end{array}$ & $\begin{array}{l}634,2 \\
576,5 \\
527,4 \\
487,2 \\
452,6 \\
630,3 \\
573,7\end{array}$ & $\begin{array}{l}5902 \\
5901 \\
5889 \\
5894 \\
5897 \\
5906 \\
5913\end{array}$ & $30^{\circ}$ \\
\hline $57 \quad 15^{\prime}$ & $\begin{array}{l}10 \\
11 \\
12 \\
13 \\
14 \\
15\end{array}$ & $\begin{array}{l}637,5 \\
579,0 \\
530,9 \\
490,1 \\
455,4 \\
424,8\end{array}$ & $\begin{array}{l}5892 \\
5887 \\
5888 \\
5889 \\
5893 \\
\mathbf{5 8 8 9}\end{array}$ & & & $\begin{array}{l}12 \\
13 \\
14\end{array}$ & $\begin{array}{l}525,1 \\
484,7 \\
450,7\end{array}$ & $\begin{array}{l}5904 \\
5904 \\
5912\end{array}$ & 30,2 \\
\hline
\end{tabular}

5. Phasendifferenzen. - Aus den in den Tabellen IV und V mitgetheilten, sowie aus meinen ubbrigen Messungen, habe ich die Phasenänderungen der senkrecht zur Einfallsebene polarisirten Componente bei der Reflexion in Luft an Quarz + derjenigen in Luft an Glas berechnet. Die interferiredden Wellenlängen im senkrecht zur Einfallsebene polarisirten Licht genügen der Gleichung:

$$
2 d \cdot \cos . \alpha+A-A^{\prime}+D_{1}+D_{2}=m \cdot \lambda \perp \text {, }
$$

wo in unserem Specialfalle $\Delta, \Delta^{\prime}, D_{1}$ und $D_{2}$ die Phasenverzögerungen bei der Reflexion in Luft an Quarz, in Glas an Luft und bei der Brechung in der die Luftschicht begrenzenden Glasfläche bedeuten. Bezeichnen wir mit $\Delta_{1}$ die Phasenverzögerung bei der Reflexion in Luft an Glas unter dem Einfallswinkel $\alpha$, und machen wir die Annahme:

$$
\Delta^{\prime}=-\Delta_{1} ; \quad D_{1}+D_{2}=0,
$$

d. h. die Annahme, dass bei innerer und äusserer Reflexion am Glase unter den entsprechenden Einfallswinkeln die senkrecht polarisirte Componente entgegengesetzt gleiche Phasenänderungen, und dass sie bei der Brechung im Glase keine Phasenänderungen erleidet, so geht die obige Gleichung für die Interferenzstreifen im senkrecht polarisirten Licht über in die Gleichung:

$$
2 d \cdot \cos . \alpha+\Delta+\Delta_{1}=m \cdot \lambda \stackrel{\perp}{ }
$$

und man erbält zur Berechnung der Summe $A+A_{1}$ :

$$
\Delta+\Delta_{1}=m . \lambda \perp-2 d \cdot \cos . \alpha \text {. }
$$


In dieser Gloichung sind $m, \lambda \perp, \alpha$ die bekannten zusammen gehörenden Grössen, wie sie in allen bisherigen Tabellen stets in gleicher Weise bezeichnet worden sind; $d$ dagegen ist die wirkliche Dicke der Luftschicht, welche ich immer aus dem unmittelbar vor oder nach dem betref-

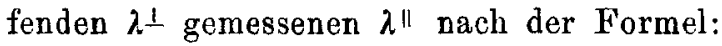

$$
d=\frac{m \cdot \lambda^{\prime \prime}}{2 \cdot \cos \cdot a}
$$

berechnet habe (und nicht etwa als das Mittel aus den verschiedenen Zahlen $d^{\prime \prime}$ ), um möglichst genau die Dicke zu erhalten, welche die Luftschicht während der Messung des betreffenden $\lambda \perp$ gehabt hatte. - Vom 14. Juli an habe ich übrigens bei der Messung der $\lambda \perp$ immer das folgende Verfahren eingeschlagen, welches die aus denselben berechneten Phasenänderungen von Aenderungen der Dicke der Luftschicht während der Messung unabhängig macht; ich habe nicht den Abstand des Streifens im senkrecht polarisirten Licht von der benachbarten Fraunhofer'schen Linie, son. dern den Abstand desselben vom nächsten Interferenzstreifen im parallel polarisirten Licht mikrometrisch bestimmt, und erhielt hieraus und aus der Lage des betreffenden parallel polarisirten Streifens die Wellenlänge des senkrecht polari. sirten Streifens. Aus den beiden zusammen gemessenen Streifen $\lambda "$ und $\lambda \perp$ wurde die Phasenänderung von $\lambda \perp$ berechnet nach der Formel:

$$
\Delta+\Delta_{1}=m \cdot \lambda^{\perp}-(m-n) \lambda^{\mu},
$$

wo je nach dem Einfallswinkel $n=0$ oder $n=1$ war, da natürlich derjenige Interferenzstreifen im parallel polarisirten Licht benutzt wurde, welcher dem senkrecht polarisirten Streifen am nächsten lag. Aenderte sich während der Messung die Dicke der Luftschicht durch Temperatureinflüsse, so wurde hierdurch die Lage der beiden Streifen im Spectrum in gleicher Weise verändert, sodass ihr Abstand ungeändert blieb.

Der Schraubenapparat ist überhaupt mehr zu Messungen der Phasendifferenzen geeignet, als zu der Untersuchung, welche der beiden Componenten eine Phasenänderung erleidet, und 
welche nicht; denn bei dieser Untersuchung handelt es sich vor allem darum, eine möglichst grosse Anzahl Zahlen $d$ zur Vergleichung zu erhalten; dies erfordert selbst bei sehr schneller Beobachtung eine Zeit von einiger Dauer, während welcher leicht Aenderungen in der Dicke und Form der Luftschicht eintreten. Dagegen kann man bei der Messung der Phasendifferenzen zur Zeit sich mit der Messung weniger Streifen begnügen, kann diese Messungen um so genauer ausführen und nach Belieben, sobald die Luftschicht nicht mehr ganz gleichmässig ist, durch Verstellen der Schrauben $s$ die vollkommene Gleichmässigkeit derselben wiederherstellen, da es in diesem Falle gar nicht darauf ankommt, dass die Dicke der Luftschicht immer dieselbe bleibt. Hierzu kommt noch der Vortheil, dass man jede halbe Stunde Sonnenschein benutzen kann.

Schliesslich setzt uns der Schraubenapparat in den Stand, die Phasenänderung jeder beliebigen 'Wellenlänge zu messen, indem man durch Verstellen der Schrauben $s$ der Luftschicht eine solche Dicke geben kann, dass im senkrecht polarisirten Licht ein Streifen in dem gewünschten Theile des Spectrums auftritt. Der Schraubenapparat kann somit dazu dienen, den Haupteinfallswinkel als Function der Wellenlänge zu untersuchen.

Meine Messungen waren zunächst für die Tabellen IV und $\mathrm{V}$ bestimmt; die aus ihnen berechneten, in den Tabellen VII und VIII mitgetheilten Phasenänderungen erreichen daher nicht die grösste von der Methode gestattete Genauigkeit. Doch tritt in denselben im grossen und ganzen hervor, dass, wie zu erwarten war, der Haupteinfallswinkel bei abnehmender Wellenlänge grösser wird.

Ich habe in den Tabellen VII und VIII die erhaltenen Resultate zunächst nach den Einfallswinkeln, innerhalb dieser nach den Versuchstagen, und innerhalb der Versuchstage nach steigenden Wellenlängen geordnet; Columne 3 enthält die Summe der Phasenänderungen der senkrecht polarisirten Componente (Phasendifferenzen der beiden Componenten) bei Reflexion in Luft an Glas und in Luft an Quarz, gemossen

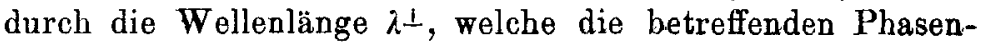


änderungen erleidet. Columne 4 gibt, ohne Rücksicht auf die Wellenlänge, die Mittel aus allen in Columne 3 für den betreffenden Einfallswinkel angegebenen Phasendifferenzen (mit Ausschluss der mit * bezeichneten); Columne 5 gibt die Zimmertemperatur während der Messung in Celsiusgraden an. Der Vollständigkeit wegen habe ich auch die ungenauen Zahlen (welche entweder nur geschätzt sind, oder wo die Luftschicht schon so ungleichmässig geworden war, dass die Streifen nicht mehr ganz schwarz erschienen) mit * bezeichnet beigefügt, sodass in den Tabellen VII und VIII alle während der Zeit vom 25. Juni bis zum 29. Juli 1887 gemessenen Wellenlängen $\lambda \perp$ im senkrecht zur Einfallsebene polarisirten Licht nebst den aus denselben berechneten Phasendifferenzen mitgetheilt sind.

\section{Tabelle VII.}

Phasendifferenzen.

Optische Axe des Quarzes senkrecht zur Einfallsebene.

\begin{tabular}{|c|c|c|c|c|c|c|c|c|c|}
\hline$\alpha$ & $\lambda \perp$ & $\frac{\Delta+\Delta_{1}}{\lambda \perp}$ & Mittel & $t$ & $\alpha$ & $\lambda \perp$ & $\frac{\Delta+A_{1}}{\lambda^{-}}$ & Mittel & $t$ \\
\hline $56^{\circ}-$ & $\begin{array}{l}479,9 \\
519,6\end{array}$ & $\begin{array}{l}0,041 \\
0,039\end{array}$ & 0,040 & $24,2^{0}$ & $57^{\circ}-$ & $\begin{array}{l}491,8 \\
573,8\end{array}$ & $\begin{array}{l}0,478 \\
0,555\end{array}$ & 0,512 & $24,4^{\circ}$ \\
\hline $56 \quad 15$ & $\begin{array}{l}478,1 \\
517,7\end{array}$ & $\begin{array}{l}0,052 \\
0,063\end{array}$ & 0,057 & & & $\begin{array}{l}499,3 \\
546,6\end{array}$ & $\begin{array}{l}0,502 \\
0,509\end{array}$ & & 24 \\
\hline $56 \quad 30$ & $\begin{array}{l}477,2 \\
516,1\end{array}$ & $\begin{array}{l}0,095 \\
0,074\end{array}$ & 0,084 & & & 506,1 & 0,514 & & 27,2 \\
\hline 5645 & $\begin{array}{l}478,8 \\
548,3\end{array}$ & $\begin{array}{l}0,271 \\
0,239\end{array}$ & 0,255 & 23 & $57 \quad 5$ & $\begin{array}{l}504,8 \\
557,6\end{array}$ & $\begin{array}{l}0,644 \\
0,716\end{array}$ & 0,680 & 24,4 \\
\hline & $519,4^{*}$ & $0,189^{*}$ & & 25,4 & $57 \quad 15$ & $\begin{array}{l}513,4 \\
563,6\end{array}$ & $\begin{array}{l}0,813 \\
0,873\end{array}$ & 0,843 & $\begin{array}{l}25 \\
26,2\end{array}$ \\
\hline 56. 50 & $\begin{array}{l}482,8 \\
559,5\end{array}$ & $\begin{array}{l}0,331 \\
0,394\end{array}$ & 0,357 & 23,4 & 5730 & $\begin{array}{l}516,9 \\
564,6\end{array}$ & $\begin{array}{l}0,928 \\
0,934\end{array}$ & 0,931 & \\
\hline $56 \quad 55$ & $\begin{array}{l}491,7 \\
565,7\end{array}$ & $\begin{array}{l}0,456 \\
0,455\end{array}$ & 0,455 & 23,7 & $57 \quad 45$ & $\begin{array}{l}514,5 \\
562,8\end{array}$ & $\begin{array}{l}0,960 \\
0,977\end{array}$ & 0,968 & 26,9 \\
\hline
\end{tabular}


Tabelle VIII.

Phasendifferenzen.

Optische Axe parallel der Einfallsebene.

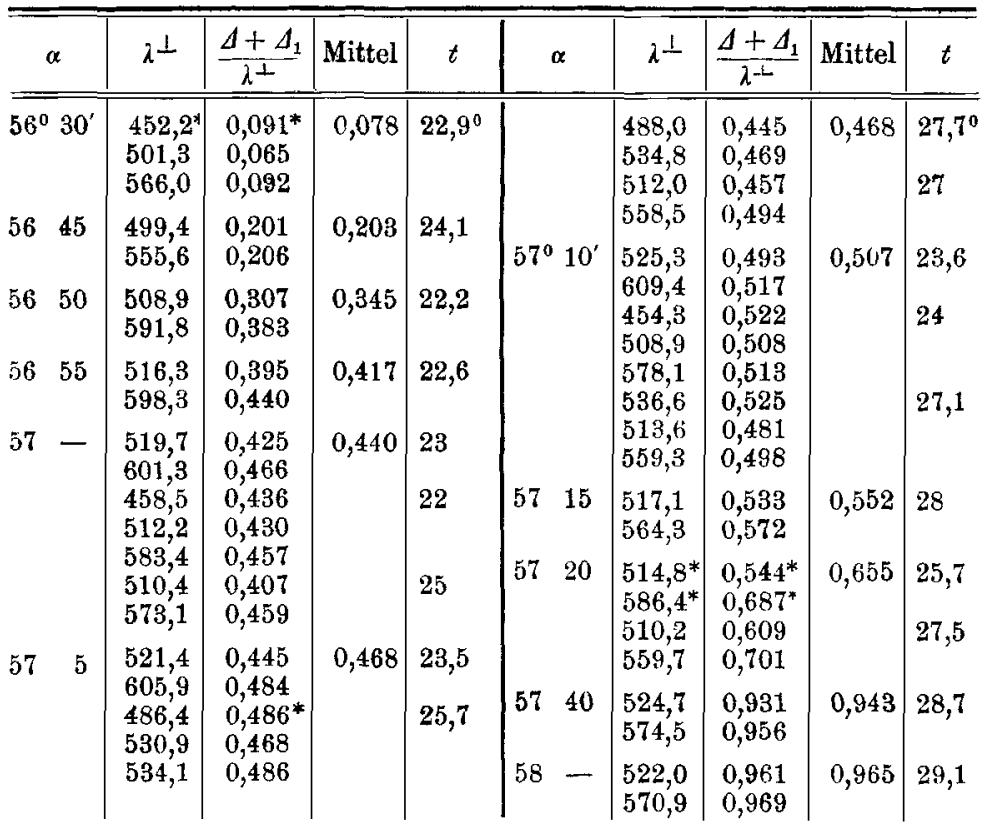

Die beiden Tabellen zeigen einen merklichen Unterschied in der Grösse des "mittleren Haupteinfallswinkels von Glas und Quarz" - wie ich den Einfallswinkel, für welchen $\left(\Delta+A_{1}\right) / \lambda .1=0.5$ ist, nennen will. Die in Columne 4 für jeden. Einfallswinkel berechneten Mittel aus den gemessenen Phasenänderungen sind sämmtlich in Tab. VII grösser, als in Tab. VIII. Man erhält in Tab. VII aus diesen Mitteln den "mittleren Haupteinfallswinkel von Glas und Quarz" $=56^{\circ} 59^{\prime}$ für den Fall, dass die optische Axe des Quarzes senkrecht zur Einfallsebene steht, dagegen in Tab. VIII für diesen Winkel den Werth $57^{\circ} 9^{\prime}$ für den Fall, dass die optische Axe der Einfallsebene parallel ist. Da dieser Unterschied nicht von der isotropen Glasfläche herrühren kann, so muss man ihn einer Verschiedenheit des Haupteinfallswinkels am Quarz, jenachdem die optische Axe senkrecht oder parallel 
zur Einfallsebene ist, zuschreiben. Die Quarzplatte und die Glasplatte sind beide im Herbst 1886, also etwa neun Monate vor diesen Versuchen geschliffen und mit dem gewöhnlichen Polirmittel (Eisenoxyd) polirt worden. Man muss daher annehmen, dass dieselben mit einer fremdartigen Oberflächenschicht behaftet waren. Diese Oberflächenschicht, welche wohl den absoluten Werth des Haupteinfallswinkels verändern mag, verdeckt also nicht die Verschiedenheit desselben je nach der Lage der optischen Axe zur Einfallsebene, trotzdem diese Verschiedenheit wegen der schwachen Doppelbrechung des Quarzes nur eine geringe ist. Dies hat seinen Grund wohl darin, dass die Oberflächenschicht in beiden Fällen den Haupteinfallswinkel in derselben Richtung verändert.

Ueber die elliptische Polarisation bei der Reflexion an doppeltbrechenden Medien ist bisher nur wenig Experimentelles veröffentlicht worden.

Jamin gibt zwar in seiner Tabelle ${ }^{1}$ die Haupteinfallswinkel verschiedener doppeltbrechender Substanzen an, doch immer für jede Substanz nur einen Werth desselben. Hr. K. Schmidtat) hat die Reflexion am Quarz nicht in Bezug auf die Doppelbrechung untersucht. Uebrigens wäre es mit den bisherigen Methoden kaum gelungen, einen Einfluss der schwachen Doppelbrechung des Quarzes auf den Hauptein. fallswinkel nachzuweisen, weil die Ungenauigkeit derselben in der Bestimmung des Einfallswinkels Beobachtungen von fünf zu fünf Minuten nicht zulässt.

Hr. E. Schenck ${ }^{3}$ ) hat bei seinen Beobachtungen über die elliptische Polarisation bei der Reflexion an zwei einaxigen, electiv absorbirenden Krystallen, am Rothgültigerz und Magnesiumplatincyanür, auf die Lage der optischen Axe Rücksicht genommen; doch sind die complicirteren Verhältnisse jener Krystalle zur Vergleichung mit Quarz nicht geeignet.

1) Jamin, Ann. de chim. et de phys. (3) 29. p. 303.1850.

2) K. Schmidt, Wied. Ann. 29. p. 451. 1886.

3) E. Schenck, Wied. Ann. 15. p. 177. 1882. 
Am besten lässt sich mein Resultat am Quarz mit den Beobachtungen Brewster's ${ }^{1}$ ) und Seebeck's ${ }^{2}$ ) über die "Polarisationswinkel" am Kalkspath und mit den Formeln, welche Neumann ${ }^{3}$ ) für den „Polarisationswinkel“ an durchsichtigen einaxigen Krystallen abgeleitet hat, vergleichen.

Brewster hat zuerst am Kalkspath ${ }^{4}$ ) eine Verschieden. heit des Polarisationswinkels je nach der Lage der reflecti. renden Fläche und der Einfallsebene zur optischen Axe beobachtet. Er findet für den Polarisationswinkel $\varphi$ einer gegebenen Fläche des Kalkspaths die empirische Gleichung:

$$
\varphi=\alpha+(\beta-\alpha) \cdot \sin ^{2} \cdot \omega,
$$

wo $\omega$ den Winkel zwischen Hauptschnitt und Einfallsebene, und wo $\alpha$ und $\beta$ die Werthe des Polarisationswinkels, wenn der Hauptschnitt parallel und senkrecht zur Einfallsebene ist, bedeuten, und wo $\beta>\alpha$ ist. D. h. der Polarisationswinkel ist ein Maximum, wenn der Hauptschnitt senkrecht zur Einfallsebene, ein Minimum, wenn der Hauptschnitt derselben parallel ist.

Seebeck hat die Versuche Brewster's am Kalkspath wiederholt und vervollständigt. Er hat durch zahlreiche Messungen an natürlichen und geschliffenen ${ }^{5}$ ) Flächen sowohl die Abhängigkeit des Polarisationswinkels vom Winkel zwischen Einfallsebene und Hauptschnitt $(\omega)$, als auch diejenige vom Winkel zwischen der reflectirenden Fläche und der optischen Axe $\left(90^{\circ}-\lambda\right)$ untersucht, und fand für den Polarisationswinkel $\varphi$, zunächst für eine gegebene Fläche des Kalkspaths:

$$
f(\varphi)=f(\alpha) \cdot \cos ^{2} \cdot \omega+f(\beta) \cdot \sin ^{2} \cdot \omega
$$

und hierauf für $\alpha$ und $\beta$ :

1) Brewster, Phil. Trans. p. 145. 1819.

2) Seebeck, Pogg. Ann. 21. p. 290. 1831.

3) Neumann, Abh. d. Berl. Acad., Math. Cl., p. 1. 1835.

4) Brewster hatte auch an einer Fläche des zweiaxigen Krystalls „chromate of lead“ (Bleichromat, Rothbleierz, krystallisirt monoklin) eine Verschiedenheit des Polarisationswinkels von $2^{\circ} 6^{r}$ je nach der Lage der Einfallsebene beobachtet.

5) Hier hatte schon Se ebeck mit dem Einfluss der vom Polirmittel herrührenden Oberflächenschicht zu kämpfen, den er schliesslich durch Anwendung von Kreide als Polirmittel auf ein Minimum beschränkte. 


$$
\begin{aligned}
& f(\alpha)=f(a) \cdot \sin ^{2} \cdot \lambda+f(c) \cdot \cos ^{2} \cdot \lambda . \\
& f(\beta)=f(b) \cdot \sin ^{2} \cdot \lambda+f(c) \cdot \cos ^{2} \cdot \lambda,
\end{aligned}
$$

wo $a, b$ und $c$ die Polarisationswinkel für eine der optischen Axe parallele Fläche, wenn die Einfallsebene parallel und senkrecht zur optischen Axe, und für eine zur optischen Axe senkrechte Fläche bedeuten. Seebeck fand eine genauere Uebereinstimmung der Formeln (1) bis (3) mit den Beobachtungen, wenn man für die Function $f$ nicht wie Brewster für Formel (1) den Winkel selbst, sondern den sinus oder cotg desselben setzt - und entschied sich zunächst für den cotg.

Durch die Gleichungen (1), (2), (3) ist der Polarisations. winkel $\varphi$ für jede beliebige Fläche $(\lambda)$ und Lage der Einfallsebene $(\omega)$ gegeben, sobald $a, b$ - die Polarisationswinkel in den zwei Hauptlagen an einer der optischen Axe parallelen, und $c$ - der Polarisationswinkel an einer zur optischen Axe senkrechten Fläche bekannt sind. Die nächste Aufgabe war nun, diese drei Grössen durch die Constanten des Krystalls, den ordentlichen $(n)$ und den ausserordentlichen Brechungsexponenten $(m)$ auszudrücken. Seebeck fand folgende empirische Formeln:
(4) $\operatorname{tg} \cdot b=n$,
5) $\operatorname{tg}, a=n \cdot \sqrt{\frac{m^{2}-1}{n^{2}-1}}$,
6) $\operatorname{tg} \cdot c=m \cdot \sqrt{\frac{n^{2}-1}{m^{2}-1}}$.

In einer späteren Abhandlung ${ }^{1}$ ) hat Seebeck versucht, diese Formeln theoretisch zu begründen, und erhielt unter Zugrundelegung der Fresnel'schen Principien (ausser der Formel: $\operatorname{tg} b=n$ ) für den Fall, dass der Hauptschnitt mit der Einfallsebene zusammenfällt, die Gleichung:

$$
\begin{gathered}
\sin ^{2} \cdot \alpha=\frac{\left(1-\mu^{2}\right) \cdot \sin ^{2} \cdot \lambda+\left(1-\nu^{2}\right) \cdot \cos ^{2} \cdot \lambda}{1-\mu^{2} \cdot \nu^{2}}, \\
\frac{1}{\nu}=n ; \quad \frac{1}{\mu}=m .
\end{gathered}
$$

In dieser Gleichung sind die Formeln (5) und (6) enthal-

1) Seebeck, Pogg. Ann. 22. p. 126. 1831. 
ten, wie man sich sofort überzeugt, wenn $\operatorname{man} \lambda=0$, und $\lambda=90^{\circ}$ setzt; sie lässt sich auch so schreiben:

$$
\sin ^{2} \cdot \alpha=\sin ^{2} \cdot a \cdot \sin ^{2} \cdot \lambda+\sin ^{2} \cdot c \cdot \cos ^{2} \cdot \lambda,
$$

woraus man erkennt, dass die Gleichung (7) nichts anderes ist, als die Gleichung (2), in welcher für die Function $f$ statt des cotg das Quadrat des sinus zu setzen ist.

Eine allgemeine Gleichung für den Polarisationswinkel bei der Reflexion an durchsichtigen einaxigen Krystallen hat zuerst $\mathrm{Neumann}{ }^{1}$ ) abgeleitet. Dieselbe geht für den Fall, dass Hauptschnitt und Einfallsebene zusammenfallen, in die Gleichung (7), - für den Fall: $\lambda=90^{\circ}, \omega=90^{\circ}$, in die Gleichung: $\operatorname{tg} b=n$ über. Die Formeln (4), (5), (6) sind somit von Neumann abgeleitet worden.

Für andere Azimuthe als $\omega=0$ bleibt bei Neumann der Polarisationswinkel durch eine ziemlich complicirte Gleichung bestimmt, die indessen bei der Berechnung keine wesentlichen Abweichungen von den einfachen empirischen Formeln (1) und (3) zeigt und mit den Messungen Seebeck's ebenfalls gut übereinstimmt. ${ }^{2}$ )

Ich stelle zunächst in Tab. IX die Polarisationswinkel $a, b, c$, welche Seebeck in den drei Hauptlagen beobachtet hat, mit denjenigen, welche er nach den Formeln (4), (5), (6) berechnet hat, zusammen. ${ }^{3}$ )

Tabelle IX. (Kalkspath.)

\begin{tabular}{|c|c|c|c|}
\hline & $a$ & $b$ & $c$ \\
\hline Berechnet: & $54^{\mathrm{u}} \quad 3,4^{\prime}$ & $58^{\circ} \quad 54,9^{\prime}$ & $60^{\circ} \quad 47,1^{\prime}$ \\
\hline Gemessen: & $\begin{array}{ll}54 & 12,4 \\
54 & 14,9\end{array}$ & $\begin{array}{ll}58 & 56 \\
58 & 56,1\end{array}$ & 33,4 \\
\hline
\end{tabular}

Ferner habe ich aus den Brechungsexponenten, welche

1) Neumann, Abh. der Berl. Acad. p. 1. 1835.

2) Neumann, l. c. p. 38 u. 39.

3) Die Zusammenstellung der übrigen von Seebeck beobachteten und berechneten Polarisationswinkel s. Pogg. Ann. 21. p. 309. 1831 und Pogg. Ann 22. p. 135. 1831. 
Rudberg ${ }^{1}$ ) für Quarz für die Fraunhofer'schen Linien $D, E, F$ angibt, nach den Formeln (4), (5), (6) die Grössen $a, b, c$ berechnet und in Tab. $\mathrm{X}$ zusammengestellt.

Tabelle X. (Quarz.)

\begin{tabular}{|c|c|c|c|c|c|}
\hline & $n$ & $m$ & $a$ & $b$ & $c$ \\
\hline$D$ & 1,51418 & 1,55328 & $57^{\circ} \quad 20,2^{\prime}$ & $57^{\circ} \quad 4,4^{\prime}$ & $\mathbf{5 6}^{0} \quad \mathbf{5 7 , 8 ^ { \prime }}$ \\
\hline$E$ & 1,54711 & 1,55631 & $57 \quad 23,2$ & $\begin{array}{ll}57 & 7,4\end{array}$ & 0,8 \\
\hline$F$ & 1,54965 & 1,55894 & $57 \quad 25,9$ & $\begin{array}{ll}57 & 9,9\end{array}$ & 3,3 \\
\hline
\end{tabular}

Dit Quarz positive Doppelbrechung besitzt, so ist für ihn $a>b>c$.

Die Differenz $a-b$ beträgt in der Tab. X etwa 16 Meine Versuche ergeben $a>b$ und die Differenz der "mitt. leren Haupteinfallswinkel von Glas und Quarz" in den bei. den Lagen der optischen Axe $=10^{\prime}$, was mit der aus den Formeln $(4),(5),(6)$ berechneten Differenz $a-b$ sehr gut übereinstimmt, wenn man bedenkt, dass wegen der Uebereinanderlagerung der Phasenänderungen an Glas und Quarz die Differenz der beobachteten ,mittleren Haupteinfallswin. kel" kleiner sein muss, als die Differenz der Haupteinfallswinkel am Quarz.

Um die absoluten Werthe der Haupteinfallswinkel am Quarz in den drei Hauptlagen zu messen und mit den in Tab. $X$ berechneten $W$ erthen zu vergleichen, müsste bei der von mir benutzten, parallel zur optischen Axe geschliffenen Fläche, und bei einer zweiten, senkrecht zur optischen Axe geschliffenen Fläche vor der Untersuchung die fremdartige Oberflächenschicht vermittelst des Wernicke'schen Gelatine. verfahrens beseitigt werden.

Berlin, im Januar 1888.

1) Rudberg, Pogg. Ann. 14. p. 52. 1828. 


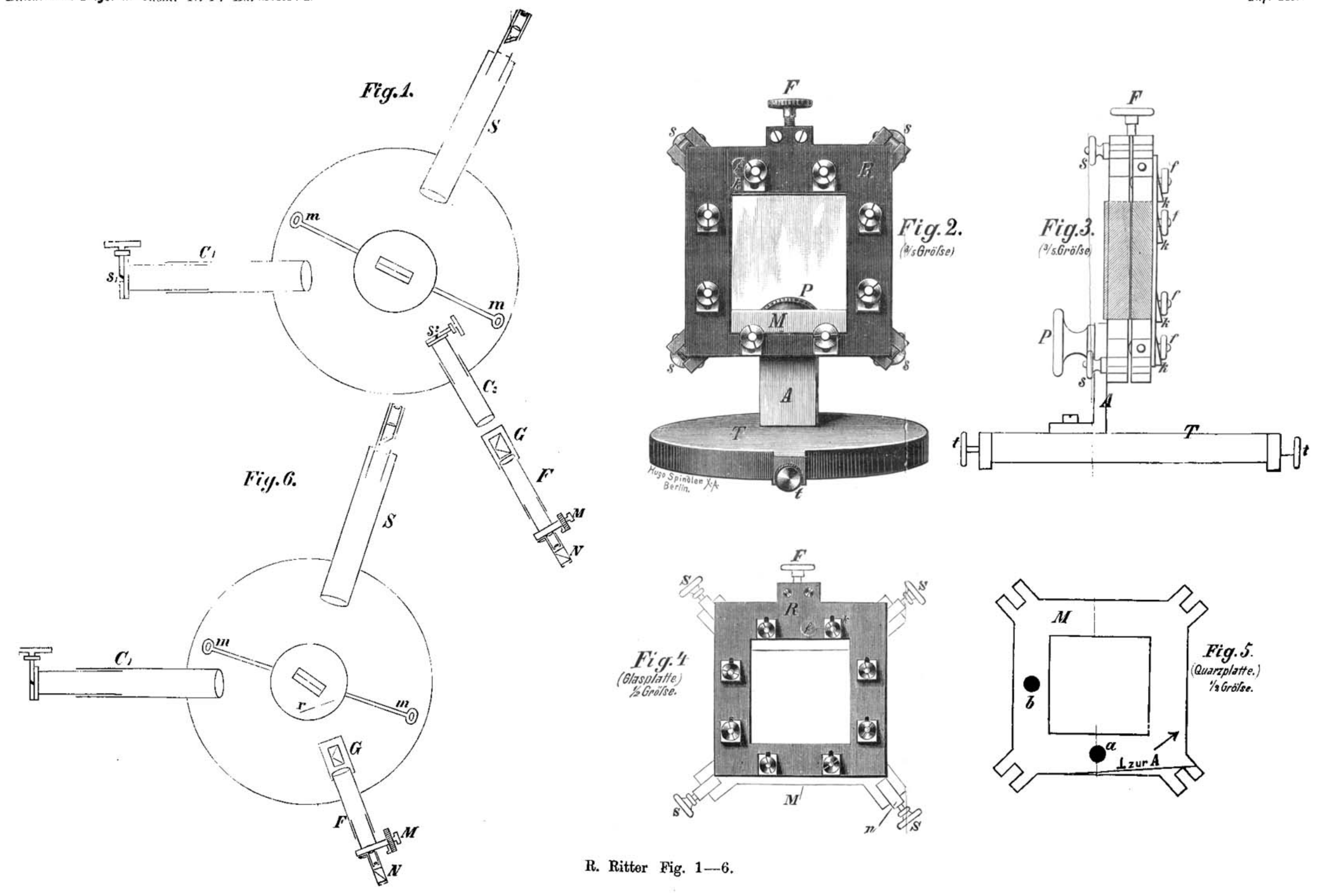

